\title{
AN $A$-BASED COFIBRANTLY GENERATED MODEL CATEGORY
}

\author{
MIGUEL OTTINA
}

\begin{abstract}
We develop a cofibrantly generated model category structure in the category of topological spaces in which weak equivalences are $A$-weak equivalences and such that the generalized $\mathrm{CW}(A)$-complexes are cofibrant objects. With this structure the exponential law turns out to be a Quillen adjunction.
\end{abstract}

\section{INTRODUCTION}

$\mathrm{CW}(A)$-complexes were introduced in 8 as topological spaces built up out of simple building blocks or cells of a certain type $A$ (called the core of the complex). The $A$ cells are cones of iterated suspensions of the space $A$. The theory of $\mathrm{CW}(A)$-complexes generalizes the classical theory of CW-complexes of J.H.C. Whitehead [12] keeping his geometric intuition and is related to the theory of $A$-cellular spaces of E.D. Farjoun [3]. There also exist other generalizations of $\mathrm{CW}$-complexes in the literature, for instance, Baues' generalization of complexes in cofibration categories [1] and Minian's categorical approach to cell complexes [7].

Much of the theory of CW $(A)$-complexes was developed in [10] where we gave a constructive and a descriptive definition and studied the topological, homological and homotopical properties of these spaces obtaining many interesting results. Among them we mention a generalization of Whitehead's theorem and a change of core theorem 8 . We also showed how the homology and homotopy groups of a $\mathrm{CW}(A)$-complex are related to those of $A$.

Apart from the homology and homotopy groups, other homotopy invariants that naturally enter the picture are the $A$-homotopy groups which are defined in a similar way to the homotopy groups but changing the spheres by iterated suspensions of the space $A$. Clearly, these generalize the homotopy groups with coefficients and, if $A$ is locally compact and Hausdorff, can be seen as the homotopy groups of a certain space of maps. Their importance in this setting is that a continuous map between $\mathrm{CW}(A)$-complexes is a homotopy equivalence if and only if it induces isomorphisms in the $A$-homotopy groups [8].

A similar result for $A$-cellular spaces had been previously proved by Farjoun in 3 . Hence, it is important and illustrative to compare both theories. This is done in section 3 of this article where we show that if $A$ is the suspension of a finite $\mathrm{CW}$-complex then the class of $A$-cellular spaces coincides with the class of spaces that have the homotopy type of a generalized $\mathrm{CW}(A)$-complex. However, the theory of $\mathrm{CW}(A)$-complexes covers a wider variety of cores $A$ since we do not require the space $A$ to have the homotopy type of a $\mathrm{CW}$-complex. On the other hand, the theory of $A$-cellular spaces, for a fixed CW-complex $A$, deals with a broader range of spaces since the class of spaces which have the homotopy

2010 Mathematics Subject Classification. 55U35.

Key words and phrases. Model Categories, Cell Structures.

Research partially supported by grant 06/M049 of SeCTyP, UNCuyo. 
type of generalized CW $(A)$-complexes is contained in the class of $A$-cellular spaces, as we shall see.

The main objetive of this article is to define a Quillen's closed model category structure 11 in the category of topological spaces, suitable for the theory of $\mathrm{CW}(A)$-complexes. Hence, the class of weak equivalences should be the class of $A$-weak equivalences, that is, the class of continuous maps which induce isomorphisms in the $A$-homotopy groups.

To this end, in section 4 we define notions of $A$-fibrations and $A$-cofibrations which together with the $A$-weak equivalences define a Quillen closed model category structure in the category of topological spaces. Moreover, we prove that this model category is cofibrantly generated. With this definitions the (generalized) $\mathrm{CW}(A)$-complexes turn out to be cofibrant objects and from the cofibrant replacement functor we obtain a $\mathrm{CW}(A)$ approximation theorem: given a topological space $X$ there exists a generalized $\mathrm{CW}(A)$ complex $Z$ together with an $A$-weak equivalence $f: Z \rightarrow X$.

Also, applying this model category structure we give a generalization of the $A$-based version of Whitehead's theorem given in [8]. In addition, we prove that the adjunction given by the exponential law is a Quillen adjunction.

This gives a theoretical framework for $\mathrm{CW}(A)$-complexes and generalizes the classical closed model category structure of pointed and path-connected topological spaces. Thus, we can regard $\mathrm{CW}(A)$-complexes as a suitable geometrically-flavoured generalization of CW-complexes.

\section{Preliminaries}

In the first part of this section we will recall briefly the main concepts on $\mathrm{CW}(A)$ complexes. In the second part, we will give some results which will be needed later.

Notations: Throughout this article we will work in the category of pointed topological spaces, hence homotopies will always be relative to the base point. $\mathbb{I}, \mathrm{C}$ and $\Sigma$ will denote the reduced cylinder, cone and suspension functors respectively. If $X$ is a (pointed) topological space, $i_{0}^{X}: X \rightarrow \mathbb{I} X$ and $i_{1}^{X}: X \rightarrow \mathbb{I} X$ will denote the standard inclusions into the bottom and the top of the cylinder respectively. Sometimes the superscript $X$ will be dropped and we will simply write $i_{0}$ and $i_{1}$ instead of $i_{0}^{X}$ and $i_{1}^{X}$. Similarly, $i_{X}$ (or simply i) will denote the inclusion $X \hookrightarrow \mathrm{C} X$.

Also, $S^{n}$ will denote the $n$-sphere, $D^{n}$ the $n$-disk and $I$ the unit interval.

In addition, if $Z$ is a space, $Z_{+}$will denote the space $Z$ with a disjoint basepoint adjoined.

Let $A$ be a fixed pointed topological space.

Definition 2.1. Let $n \in \mathbb{N}$. We say that a (pointed) space $X$ is obtained from a (pointed) space $B$ by attaching $A$ - $n$-cells if there exists a pushout diagram

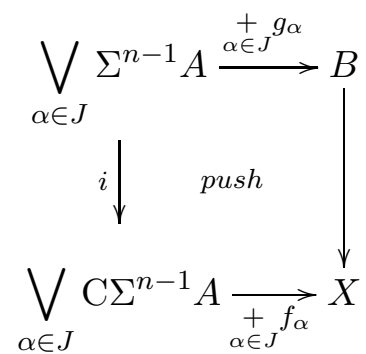


where $J$ is any set.

The $A$-cells are the images of the maps $f_{\alpha}$. The maps $g_{\alpha}$ are the attaching maps of the cells, and the maps $f_{\alpha}$ are its characteristic maps. We say that $X$ is obtained from $B$ by attaching A-0-cells if $X=B \vee \bigvee_{\alpha \in J} A$.

For example, the reduced cone $\mathrm{C} A$ of $A$ is obtained from $A$ by attaching an $A$-1-cell. In particular, $D^{2}$ is obtained from $D^{1}$ by attaching a $D^{1}-1$-cell. Also, the reduced suspension $\Sigma A$ can be obtained from the singleton $*$ by attaching an $A$-1-cell.

Note that attaching an $S^{0}$-n-cell is the same as attaching an $n$-cell in the usual sense (but preserving base points), and that attaching an $S^{m}$-n-cell means attaching an $(m+n)$-cell in the usual sense (preserving base points).

Definition 2.2. A $C W(A)$-complex structure on $X$, is a sequence of spaces

$$
*=X^{-1}, X^{0}, X^{1}, \ldots, X^{n}, \ldots
$$

such that, for $n \in \mathbb{N}_{0}, X^{n}$ is obtained from $X^{n-1}$ by attaching $A$ - $n$-cells, and $X$ is the colimit of the diagram

$$
*=X^{-1} \rightarrow X^{0} \rightarrow X^{1} \rightarrow \ldots \rightarrow X^{n} \rightarrow \ldots
$$

We say that the space $X$ is a $C W(A)$-complex if it admits some $\mathrm{CW}(A)$-complex structure. In this case, the space $A$ will be called the core or the base space of the structure.

\section{Examples 2.3.}

(1) A $\mathrm{CW}\left(S^{0}\right)$-complex is just a pointed $\mathrm{CW}$-complex with all adjunction maps of cells of positive dimension preserving base points. Clearly, any pointed and pathconnected CW-complex has the homotopy type of a $\mathrm{CW}\left(S^{0}\right)$-complex.

Similarly, for all $n \in \mathbb{N}$ a $\mathrm{CW}\left(S^{n}\right)$-complex is a pointed $\mathrm{CW}$-complex with no cells of dimension less than $n$, apart from the base point. Also, any $n$-connected $\mathrm{CW}$-complex has the homotopy type of a $\mathrm{CW}\left(S^{n}\right)$-complex.

(2) The space $D^{n}$ admits several different $\mathrm{CW}\left(D^{1}\right)$-complex structures. For instance, we can take $X^{r}=D^{r+1}$ for $0 \leq r \leq n-1$ since $\mathrm{C} D^{r}$ is homeomorphic to $D^{r+1}$ and $\Sigma^{r-1} D^{1}$ is homeomorphic to $D^{r}$ for all $r$. We may also define

$$
X^{0}=\ldots=X^{n-2}=* \quad \text { and } \quad X^{n-1}=D^{n}
$$

since there is a pushout

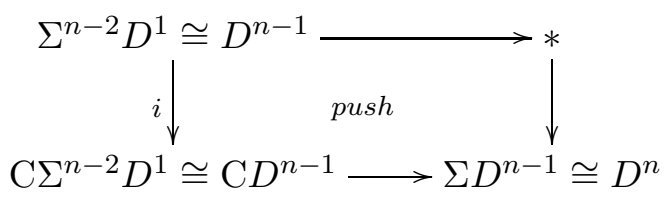

In [8] we analyzed the change from a core $A$ to a core $B$ by means of a map $\alpha: A \rightarrow B$ and obtained the following result.

Theorem 2.4. Let $A$ and $B$ be pointed topological spaces with closed base points, let $X$ be a $C W(A)$-complex and let $\alpha: A \rightarrow B$ and $\beta: B \rightarrow A$ be continuous maps.

(a) If $\beta \alpha=\operatorname{Id}_{A}$, then there exist a $C W(B)$-complex $Y$ and maps $\varphi: X \rightarrow Y$ and $\psi: Y \rightarrow X$ such that $\psi \varphi=\operatorname{Id}_{X}$.

(b) If $\beta$ is a homotopy equivalence, then there exist a $C W(B)$-complex $Y$ and a homotopy equivalence $\varphi: X \rightarrow Y$. 
(c) If $\beta \alpha=\operatorname{Id}_{A}$ and $\alpha \beta \simeq \operatorname{Id}_{B}$ then there exist a $C W(B)$-complex $Y$ and maps $\varphi: X \rightarrow Y$ and $\psi: Y \rightarrow X$ such that $\psi \varphi=\operatorname{Id}_{X}$ and $\varphi \psi \simeq \operatorname{Id}_{Y}$.

In particular, when the core $A$ is contractible, all $\mathrm{CW}(A)$-complexes are also contractible.

Let $X$ be a (pointed) topological space. Recall that, for $r \in \mathbb{N}_{0}$, the sets $\pi_{r}^{A}(X)$ are defined by $\pi_{r}^{A}(X)=\left[\Sigma^{r} A, X\right]$, this is the homotopy classes of (pointed) maps from $\Sigma^{r} A$ to $X$. Similarly, for $B \subseteq X$ and $r \in \mathbb{N}$ one defines $\pi_{r}^{A}(X, B)=\left[\left(\mathrm{C} \Sigma^{r-1} A, \Sigma^{r-1} A\right),(X, B)\right]$.

We say that a space $X$ is $A$-n-connected if $\pi_{r}^{A}(X)=0$ for $0 \leq r \leq n$.

We also say that a continuous map $f: X \rightarrow Y$ between (pointed) topological spaces is an $A$-weak equivalence if it induces isomorphisms $f_{*}: \pi_{n}^{A}(X) \rightarrow \pi_{n}^{A}(Y)$ for all $n \in \mathbb{N}_{0}$.

Remark 2.5. Let $X$ and $Y$ be pointed topological spaces and let $f: X \rightarrow Y$ be a continuous map. Let $\mathcal{O}$ denote the forgetful functor from the category of pointed topological spaces to the category of topological spaces. Note that if $\mathcal{O}(f)$ is a weak homotopy equivalence then $f$ is an $S^{0}$-weak equivalence.

The converse of this implication holds if $X$ is path-connected but does not hold in general as the following example shows. Let $X=S^{0}$ and let $Y=\left\{(x, y) \in \mathbb{R}^{2} /(x+2)^{2}+y^{2}=\right.$ $1\} \cup\{(1,0)\}$ with the subspace topology with respect to $\mathbb{R}^{2}$ and with $(1,0)$ as the base point. Let $f: X \rightarrow Y$ be defined by $f(x)=(x, 0)$. The map $f$ is an $S^{0}$-weak equivalence but not a weak homotopy equivalence.

Moreover, it is easy to prove that a continuous map $f: X \rightarrow Y$ is an $S^{0}$-weak equivalence if and only if $\mathcal{O}(f)_{*}: \pi_{0}(X) \rightarrow \pi_{0}(Y)$ is a bijection and the map that $\mathcal{O}(f)$ induces between the path components of $X$ and $Y$ that contain the base points is a weak homotopy equivalence.

In [8] we gave the following generalization of Whitehead's theorem. In the next section we will compare this result with a similar one of E.D.Farjoun [3].

Theorem 2.6. Let $X$ and $Y$ be $C W(A)$-complexes and let $f: X \rightarrow Y$ be a continuous map. Then $f$ is a homotopy equivalence if and only if it is an $A$-weak equivalence.

We also say that a space $X$ is a generalized $C W(A)$-complex if it is obtained from the singleton $*$ by attaching $A$-cells in countably many steps, allowing cells of any dimension to be attached in any step. More precisely, we have the following definition.

Definition 2.7. Let $X, A$ and $B$ be (pointed) topological spaces. We say that $X$ is obtained from $B$ by attaching $A$-cells if there exists a pushout

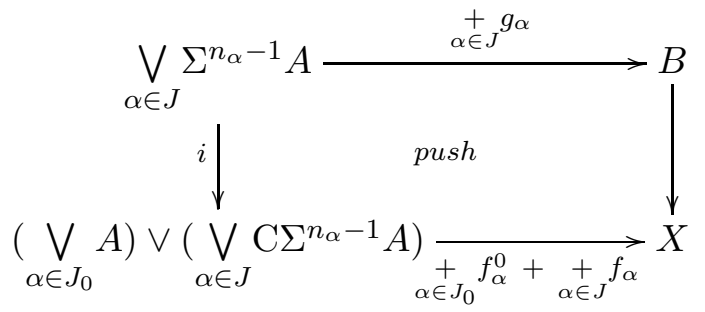

where $J_{0}$ and $J$ are sets and where $n_{\alpha} \in \mathbb{N}$ for all $\alpha \in J$.

We say that $X$ is a generalized $C W(A)$-complex if $X$ is the colimit of a diagram

$$
*=X^{0} \rightarrow X^{1} \rightarrow X^{2} \rightarrow \ldots \rightarrow X^{n} \rightarrow \ldots
$$

where $X^{n}$ is obtained from $X^{n-1}$ by attaching $A$-cells. 
We call $X^{n}$ the $n$-th layer of $X$.

We also say that a (pointed) topological pair $(X, B)$ is a relative $C W(A)$-complex if $X$ is the colimit of a diagram

$$
B=X^{-1} \rightarrow X^{0} \rightarrow X^{1} \rightarrow X^{2} \rightarrow \ldots \rightarrow X^{n} \rightarrow \ldots
$$

where $X^{n}$ is obtained from $X^{n-1}$ by attaching $A$-n-cells.

Finally, we say that a (pointed) topological pair $(X, B)$ is a generalized relative $C W(A)$ complex if $X$ is the colimit of a diagram

$$
B=X^{0} \rightarrow X^{1} \rightarrow X^{2} \rightarrow \ldots \rightarrow X^{n} \rightarrow \ldots
$$

where $X^{n}$ is obtained from $X^{n-1}$ by attaching $A$-cells.

A generalized $\mathrm{CW}\left(S^{0}\right)$-complex will be called a generalized $\mathrm{CW}$-complex. It is easy to prove that a generalized $\mathrm{CW}$-complex has the homotopy type of a $\mathrm{CW}$-complex. Moreover, we prove in [8] that if $A$ is any $\mathrm{CW}$-complex then a generalized $\mathrm{CW}(A)$-complex has the homotopy type of a $\mathrm{CW}$-complex. However, a generalized $\mathrm{CW}(A)$-complex does not necessarily have the homotopy type of a $\mathrm{CW}(A)$-complex (see [9], example 3.10).

Now we will develop some results which will be needed later. We start by studying under which conditions the reduced cylinder of a $\mathrm{CW}(A)$-complex is again a $\mathrm{CW}(A)$-complex.

Lemma 2.8. Let $\nu: S^{1} \rightarrow S^{1} \vee S^{1}$ be the usual map inducing the comultiplication in $S^{1}$. Then there is a pushout

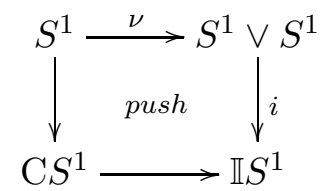

where $i\left(S^{1} \vee S^{1}\right)=i_{0}\left(S^{1}\right) \vee i_{1}\left(S^{1}\right)$.

Proof. Note that the pushout of the diagram

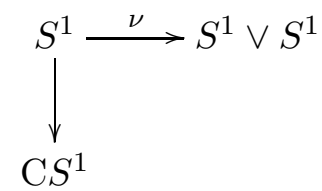

is $D^{2} /\{(-1,0),(1,0)\}$. There are homeomorphisms

$$
\mathbb{I} S^{1}=S^{1} \times I /(\{(1,0)\} \times I) \cong(I \times I) /(\{0,1\} \times I) \cong D^{2} /\{(-1,0),(1,0)\}
$$

and hence, the result follows.

Proposition 2.9. Let $A^{\prime}$ be a locally compact and Hausdorff space and let $A=\Sigma A^{\prime}$. Let $X$ be a $C W(A)$-complex. Then the reduced cylinder $\mathbb{I} X$ is a $C W(A)$-complex. Moreover, $i_{0}(X)$ and $i_{1}(X)$ are $C W(A)$-subcomplexes of $\mathbb{I} X$.

Proof. For $n \in \mathbb{N}$ let $J_{n}$ be an index set for the $A$ - $n$-cells of $X$. We proceed by induction in the $A$-skeletons of $X$. For the initial case we have that $X^{0}=\bigvee_{\alpha \in J_{0}} A$. Then $\mathbb{I} X^{0}=\bigvee_{\alpha \in J_{0}} \mathbb{I} A$. 
But $\mathbb{I} A$ is a $\mathrm{CW}(A)$-complex since applying $-\wedge A^{\prime}$ to the pushout of the previous lemma gives a pushout

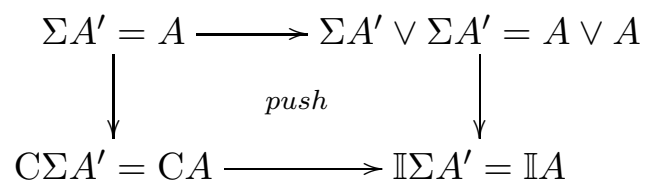

since $\mathrm{C} S^{1} \wedge A^{\prime} \cong I \wedge S^{1} \wedge A^{\prime} \cong I \wedge \Sigma A^{\prime} \cong \mathrm{C} \Sigma A^{\prime}$ and $\mathbb{I} S^{1} \wedge A^{\prime} \cong I_{+} \wedge S^{1} \wedge A^{\prime} \cong I_{+} \wedge \Sigma A^{\prime} \cong \mathbb{I} \Sigma A^{\prime}$. Moreover, $i_{0}(A)$ and $i_{1}(A)$ are $\mathrm{CW}(A)$-subcomplexes of $\mathbb{I} A$. Hence, $\mathbb{I} X^{0}$ is a $\mathrm{CW}(A)$ complex and $i_{0}\left(X^{0}\right)$ and $i_{1}\left(X^{0}\right)$ are $\mathrm{CW}(A)$-subcomplexes of $\mathbb{I} X^{0}$.

Now suppose that $\mathbb{I} X^{n-1}$ is a $\mathrm{CW}(A)$-complex and that $i_{0}\left(X^{n-1}\right)$ and $i_{1}\left(X^{n-1}\right)$ are $\mathrm{CW}(A)$-subcomplexes of $\mathbb{I} X^{n-1}$. We consider
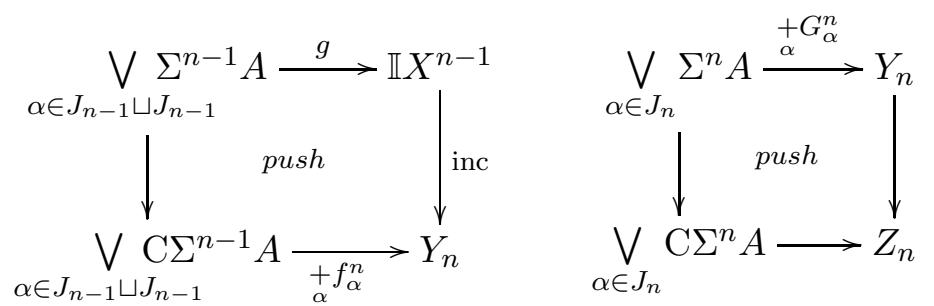

with $g=\left(\begin{array}{r}+i_{0} g_{\alpha}^{n} \\ \alpha \in J_{n-1}\end{array}\right)+\left(\begin{array}{r}+i_{1} g_{\alpha}^{n} \\ \alpha \in J_{n-1}\end{array}\right)$ where $\left(g_{\alpha}^{n}\right)_{\alpha \in J_{n-1}}$ are the adjunction maps of the $A$ - $n$-cells of $X$, and where the maps $G_{\alpha}, \alpha \in J_{n}$, are defined as the composition

$$
\Sigma\left(\Sigma^{n-1} A\right) \longrightarrow \mathrm{C}\left(\Sigma^{n-1} A\right) \bigcup_{\Sigma^{n-1} A} \mathbb{I}\left(\Sigma^{n-1} A\right) \bigcup_{\Sigma^{n-1} A} \mathrm{C}\left(\Sigma^{n-1} A\right) \stackrel{f_{\alpha}^{n} \cup F_{\alpha} \cup f_{\alpha}^{n}}{\longrightarrow} Y_{n}
$$

where the first map is the standard homeomorphism and where $F_{\alpha}$ is the composition

$$
\mathbb{I}\left(\sum^{n-1} A\right) \stackrel{\mathbb{I} g_{\alpha}^{n}}{\longrightarrow} \mathbb{I} X^{n-1} \stackrel{\text { inc }}{\longrightarrow} Y_{n}
$$

We wish to prove that $Z_{n}$ is homeomorphic to $\mathbb{I} X^{n}$. Note that

$$
Y_{n}=X^{n} \bigcup_{X^{n-1}} \mathbb{I} X^{n-1} \bigcup_{X^{n-1}} X^{n}
$$

We have that

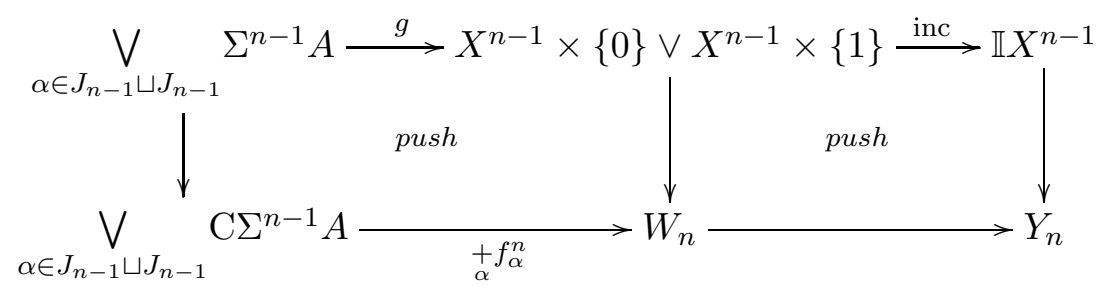

and clearly $W_{n}=X^{n} \times\{0\} \vee X^{n} \times\{1\}$.

Now, the homeomorphism $\Sigma\left(\Sigma^{n-1} A\right) \longrightarrow \mathrm{C}\left(\Sigma^{n-1} A\right) \bigcup_{\Sigma^{n-1} A} \mathbb{I}\left(\Sigma^{n-1} A\right) \bigcup_{\Sigma^{n-1} A} \mathrm{C}\left(\Sigma^{n-1} A\right)$ extends to a homeomorphism $\mathrm{C}\left(\Sigma^{n} A\right) \longrightarrow \mathbb{I} \mathrm{C}\left(\Sigma^{n-1} A\right)$. Indeed, this follows applying $-\wedge A$ to the homeomorphism of topological pairs $\psi:\left(D^{n+1}, S^{n}\right) \rightarrow\left(\mathbb{I} D^{n}, D^{n} \cup_{S^{n-1}} \mathbb{I} S^{n-1} \underset{S^{n-1}}{\cup} D^{n}\right)$ 
Then, we have

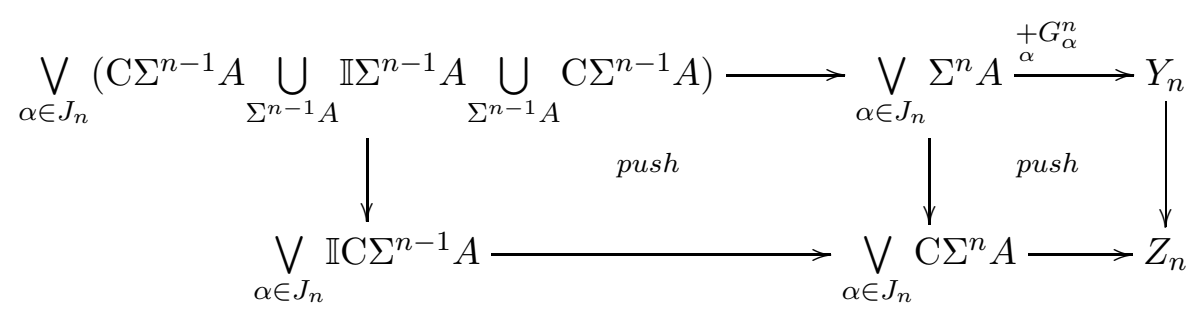

Note that the first square is a pushout since it commutes and its two horizontal arrows are homeomorphisms.

Note also that the top horizontal composition is $\underset{\alpha}{+}\left(f_{\alpha}^{n} \cup F_{\alpha} \cup f_{\alpha}^{n}\right)$ and that $Z_{n}=\mathbb{I} X^{n}$ since $F_{\alpha}=$ inc $\circ \mathbb{I} g_{\alpha}^{n}$. The result follows.

The following two lemmas will be used in section 4 .

Lemma 2.10. Let $B$ be a (pointed) topological space. Then there exist homeomorphisms of pointed topological pairs:

(a) $\left(\mathbb{I} \mathrm{C} B, i_{0}(\mathrm{C} B)\right) \cong\left(\mathbb{I} \mathrm{C} B, i_{0}(\mathrm{C} B) \cup \mathbb{I} B\right)$

(b) $\left(\mathbb{I} \mathrm{C} B, i_{0}(\mathrm{C} B) \cup \mathbb{I}(i(B)) \cup i_{1}(\mathrm{C} B)\right) \cong(\mathrm{C} \Sigma B, \Sigma B)$

(c) $\left.\left(\mathbb{I} \mathbb{I} B, i_{0}(\mathbb{I} B)\right) \cong\left(\mathbb{I} \mathbb{I} B, \mathbb{I}\left(i_{0}(B)\right) \cup i_{0}(\mathbb{I} B)\right)\right)$

Proof. Recall that if $Z$ is a topological space, $Z_{+}$denotes the space $Z$ with a disjoint basepoint adjoined. Also, in what follows 0 will be the base point of the spaces $I$ and $\{0,1\} \subseteq I$ and the point $(1,0) \in \mathbb{R}^{2}$ will be the base point of the disk $D^{2}$.

Let $T=\left\{(x, y) \in S^{1} / y \leq 0\right\}$.

(a) Note that the topological pairs $\left(I_{+} \wedge I,\{0\}_{+} \wedge I\right)$ and $\left(I_{+} \wedge I,\left(\{0\}_{+} \wedge I\right) \cup\left(I_{+} \wedge\{0,1\}\right)\right)$ are homeomorphic since both of them are homeomorphic to $\left(D^{2}, T\right)$. Hence, item (a) follows taking smash product with $B$.

(b) Note that the topological pairs $\left(I_{+} \wedge I,\left(\{0\}_{+} \wedge I\right) \cup\left(I_{+} \wedge\{0,1\}\right) \cup\left(\{1\}_{+} \wedge I\right)\right)$ and $\left(I \wedge S^{1},\{0,1\} \wedge S^{1}\right)$ are homeomorphic since both of them are homeomorphic to $\left(D^{2}, S^{1}\right)$. Hence, item (b) follows taking smash product with $B$.

(c) Note that the topological pairs $\left(I_{+} \wedge I_{+},\{0\}_{+} \wedge I_{+}\right)$and $\left(I_{+} \wedge I_{+},\left(\{0\}_{+} \wedge I_{+}\right) \cup\left(I_{+} \wedge\right.\right.$ $\left.\left.\{0\}_{+}\right)\right)$are homeomorphic since both of them are homeomorphic to $\left(D_{+}^{2}, T_{+}\right)$. Hence, item (c) follows taking smash product with $B$.

Lemma 2.11. Let $B$ be a topological space. We define in $\mathbb{I C} B$ an equivalence relation as follows. If $x, x^{\prime} \in \mathrm{C} B$ and $t, t^{\prime} \in I$ we say that $(x, t) \sim\left(x^{\prime}, t^{\prime}\right)$ if and only if $x \in B$ and $x=x^{\prime}$. Let $q: \mathbb{I C} B \rightarrow \mathbb{I C} B / \sim$ be the quotient map. Let $\Sigma_{+} B=\left\{[b, t] \in \Sigma B / t \geq \frac{1}{2}\right\}$ and $\Sigma_{-} B=\left\{[b, t] \in \Sigma B / t \leq \frac{1}{2}\right\}$. Then there exists a homeomorphism $\varphi: \mathbb{I C} B / \sim \rightarrow \mathrm{C} \Sigma B$ such that $\varphi\left(q\left(i_{0}(\mathrm{C} B)\right)\right)=\Sigma_{-} B$ and $\varphi\left(q\left(i_{1}(\mathrm{C} B)\right)\right)=\Sigma_{+} B$.

Proof. Let $\sim_{0}$ be the equivalence relation in $\mathbb{I C} S^{0}$ defined as the one above, that is, if $x, x^{\prime} \in \mathrm{C} S^{0}$ and $t, t^{\prime} \in I$, then $(x, t) \sim_{0}\left(x^{\prime}, t^{\prime}\right)$ if and only if $x \in S^{0}$ and $x=x^{\prime}$. There are homeomorphisms

$$
\begin{aligned}
\mathbb{I C} B / \sim & \cong I_{+} \wedge I \wedge B / \sim \cong I_{+} \wedge I \wedge S^{0} \wedge B / \sim \cong \mathbb{I C} S^{0} \wedge B / \sim \cong \mathbb{I C} S^{0} / \sim_{0} \wedge B \cong \\
& \cong \mathbb{I} D^{1} / \sim_{0} \wedge B \cong D^{2} \wedge B \cong \mathrm{C} S^{1} \wedge B \cong I \wedge S^{1} \wedge B \cong \mathrm{C} \Sigma B .
\end{aligned}
$$

The result follows. 


\section{Comparison between CW $(A)$-Complexes and Dror Farjoun's $A$-Cellular SPACES}

In this section we will compare $\mathrm{CW}(A)$-complexes [10] with $A$-cellular spaces $[3$. We begin by recalling Dror Farjoun's definition of $A$-cellular spaces.

Definition 3.1. Let $A$ be a (pointed) topological space having the homotopy type of a CW-complex. The class of A-cellular spaces is the smallest class of topological spaces that contains the space $A$ and is closed under homotopy equivalences and pointed homotopy colimits.

Note that in Dror Farjoun's setting the space $A$ is required to have the homotopy type of a CW-complex, while we allow $A$ to be any space. Hence, our results are more general to a certain extent. As an illustrative example, let us compare our version of the $A$-Whitehead theorem (8], theorem 5.10) with the one given in 3 (theorem E.1 of chapter 2). If $A$ is a suspension of a finite CW-complex then both versions coincide (cf. proposition 3.2). But our version covers a wider variety of cores $A$, while Dror Farjoun's one, for a fixed $\mathrm{CW}$-complex $A$, deals with a broader range of spaces since the class of $\mathrm{CW}(A)$-complexes is strictly contained in the class of $A$-cellular spaces as we shall see.

Until the end of this section, $A$ will denote a fixed pointed topological space having the homotopy type of a CW-complex.

Before going on, we fix the following notation.

- A-Cel will denote the class of $A$-cellular spaces.

- $\mathcal{C W}(A)$ will denote the class of $\mathrm{CW}(A)$-complexes.

- $\mathcal{H} \mathcal{T C W}(A)$ will denote the class of spaces having the homotopy type of a $\mathrm{CW}(A)$ complex.

- $\mathcal{C W}(A)$-gen will denote the class of generalized $\mathrm{CW}(A)$-complexes.

- $\mathcal{H} \mathcal{T C W}(A)$-gen will denote the class of spaces having the homotopy type of a generalized $\mathrm{CW}(A)$-complex.

We aim to compare these five classes of topological spaces. It is clear that

$$
\mathcal{C W}(A) \subseteq \mathcal{C W}(A) \text {-gen } \subseteq \mathcal{H} \mathcal{T C W}(A) \text {-gen }
$$

and

$$
\mathcal{C W}(A) \subseteq \mathcal{H} \mathcal{T C W}(A) \subseteq \mathcal{H} \mathcal{T C W}(A) \text {-gen }
$$

Also, since the constructions of $\mathrm{CW}(A)$-complexes and generalized $\mathrm{CW}(A)$-complexes can be rephrased in terms of homotopy colimits we get that

$$
\mathcal{H} \mathcal{T C W}(A) \text {-gen } \subseteq \text { A-Cel } .
$$

Now, let $C W_{A}$ denote the $A$-cellular approximation functor described in [3]. From section 2.E of that work, we conclude that if $A$ is the suspension of a finite $\mathrm{CW}$-complex and $Z$ is a topological space which has the homotopy type of a CW-complex then $C W_{A}(Z)$ can be obtained by an inductive process of attaching $A$-cells. Therefore, $C W_{A}(Z)$ is a generalized CW $(A)$-complex. Then, if $X$ is an $A$-cellular space we obtain that $X$ is homotopy equivalent to $C W_{A}(X)$ and hence $X$ has the homotopy type of a generalized $\mathrm{CW}(A)$-complex. Summing up, we have proved the following.

Proposition 3.2. If $A$ is the suspension of a finite $C W$-complex then

$$
\mathcal{H} \mathcal{T C W}(A) \text {-gen }=A-\mathcal{C} \text { el } .
$$


However, the other inclusions are strict in general. Indeed, in example 3.10 of 9 ] we exhibit a generalized $\mathrm{CW}(A)$-complex which does not have the homotopy type of a $\mathrm{CW}(A)$ complex. This shows that $\mathcal{C W}(A) \subsetneq \mathcal{C W}(A)$-gen and $\mathcal{H} \mathcal{T C W}(A) \subsetneq \mathcal{H} \mathcal{C} \mathcal{W}(A)$-gen. On the other hand, the inclusions $\mathcal{C W}(A) \subseteq \mathcal{H} \mathcal{T C W}(A)$ and $\mathcal{C W}(A)$-gen $\subseteq \mathcal{H} \mathcal{T C W}(A)$-gen are easily seen to be strict.

\section{An $A$-based cofibrantly generated model Category}

In this section we will define appropriate notions of $A$-fibrations and $A$-cofibrations and prove that together with the $A$-weak equivalences they define a closed model category structure in the category of pointed topological spaces. Moreover, we will prove that this model category is cofibrantly generated.

Recall that if $\mathcal{C}$ is a category and $\mathcal{I}$ is a set of maps in $\mathcal{C}$ then the class of $\mathcal{I}$-injectives is the class of maps that have the right lifting property (RLP) with respect to every map in $\mathcal{I}$ and is denoted by $\mathcal{I}$-inj. Also, the class of $\mathcal{I}$-cofibrations is the class of maps that have the left lifting property (LLP) with respect to every map in $\mathcal{I}$-inj and is denoted by $\mathcal{I}$-cof.

For the rest of this section, let $A$ be a fixed pointed topological space.

Let

$$
\mathcal{I}_{A}=\left\{\Sigma^{n-1} A \hookrightarrow \mathrm{C} \Sigma^{n-1} A / n \in \mathbb{N}\right\} \cup\left\{i_{0}: \mathbb{I}^{n-1} A \rightarrow \mathbb{I}^{n} A / n \in \mathbb{N}\right\} \cup\{* \rightarrow A\}
$$

and let

$$
\mathcal{J}_{A}=\left\{i_{0}: \mathbb{I}^{n-1} A \rightarrow \mathbb{I}^{n} A / n \in \mathbb{N}\right\} \cup\left\{i_{0}: \mathrm{C} \Sigma^{n-1} A \rightarrow \mathbb{I C} \Sigma^{n-1} A / n \in \mathbb{N}\right\}
$$

where $\mathbb{I}^{0}$ and $\Sigma^{0}$ denote the identity functors.

The set $\mathcal{I}_{A}$ will be the set of generating cofibrations and the set $\mathcal{J}_{A}$ will be the set of generating trivial cofibrations of our model category, as we shall see.

Definition 4.1. Let $E$ and $B$ be (pointed) topological spaces and let $p: E \rightarrow B$ be a continuous map. We say that $p$ is an $A$-fibration if it has the homotopy lifting property (HLP) with respect to the spaces $A$ and $\mathrm{C} \Sigma^{n-1} A, n \in \mathbb{N}$, and $\mathbb{I}^{n} A, n \in \mathbb{N}$.

The class of $A$-fibrations will be denoted by Fib.

Note that Fib $=\mathcal{J}_{A \text {-inj. }}$

Let WE be the class of $A$-weak equivalences and let Cof be the class of maps which have the left lifting property with respect to the class Fib $\cap$ WE. The maps in the class Cof will be called $A$-cofibrations. If $X$ is a topological space such that the map $* \rightarrow X$ is an $A$-cofibration, we will say that $X$ is an $A$-cofibrant space.

The following proposition shows that $S^{0}$-fibrations are just Serre fibrations.

Proposition 4.2. Let $X$ and $Y$ be pointed topological spaces and let $p: X \rightarrow Y$ be a continuous map. Let $\mathcal{O}$ denote the forgetful functor from the category of pointed topological spaces to the category of topological spaces. Then the map $p$ is an $S^{0}$-fibration if and only if $\mathcal{O}(p)$ is a Serre fibration.

Proof. Suppose first that $p$ is an $S^{0}$-fibration. By definition, $p$ has the HLP with respect to $\mathbb{I}^{n} S^{0}$ for all $n \in \mathbb{N}_{0}$. Note that, for $n \in \mathbb{N}$, II $\mathbb{I}^{n} S^{0}$ is equal to $I_{+}^{n}$ which is homeomorphic to $D_{+}^{n}$ and $\mathbb{I}^{0} S^{0}=S^{0}$ is homeomorphic to $D_{+}^{0}$. It follows that $\mathcal{O}(p)$ has the (unbased) homotopy lifting property with respect to $D^{n}$ for all $n \in \mathbb{N}_{0}$. Hence, $\mathcal{O}(p)$ is a Serre fibration.

Conversely, if the map $\mathcal{O}(p)$ is a Serre fibration then it has the homotopy lifting property with respect to $D^{n}$ for all $n \in \mathbb{N}_{0}$. Since $\mathbb{I}^{n} S^{0}$ is homeomorphic to $D_{+}^{n}$ for all $n \in \mathbb{N}_{0}$ it 
follows that the map $p$ has the HLP with respect to $\mathbb{I}^{n} S^{0}$ for all $n \in \mathbb{N}_{0}$. It remains to prove that $p$ has the HLP with respect to $\mathrm{C} \Sigma^{n-1} S^{0}$ for all $n \in \mathbb{N}$.

Since $\mathrm{C} \Sigma^{n-1} S^{0}$ is homeomorphic to $D^{n}$ which is homeomorphic to $\mathbb{I}^{n-1} I$, it suffices to prove that $p$ has the RLP with respect to the inclusion $i_{0}: \mathbb{I}^{n-1} I \rightarrow \mathbb{I}^{n} I$ for all $n \in \mathbb{N}$.

Let $n \in \mathbb{N}$ and let $f: \mathbb{I}^{n-1} I \rightarrow X$ and $H: \mathbb{I}^{n} I \rightarrow Y$ be continuous maps such that $H i_{0}=p f$. For $j \in \mathbb{N}$ let $q_{j}: I^{j} \rightarrow \mathbb{I}^{j-1} I \cong I^{j} /\left(\{0\} \times I^{j-1}\right)$ be the quotient map. Let $x_{0}$ and $y_{0}$ be the base points of $X$ and $Y$ respectively and let $F:\left(I^{n} \times\{0\}\right) \cup\left(\{0\} \times I^{n}\right) \rightarrow X$ be defined by $F(z, 0)=f\left(q_{n}(z)\right)$ and $F(0, z)=x_{0}$ for $z \in I^{n}$. Clearly, the map $F$ is well-defined and continuous.

There is a commutative diagram of unpointed spaces and maps

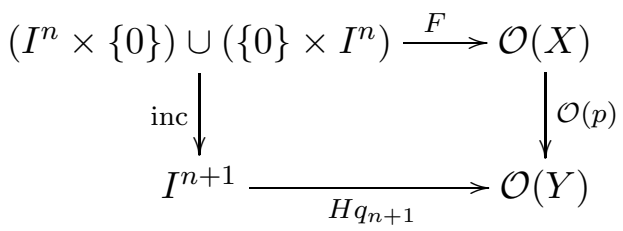

Now, since the topological pairs $\left(I^{n+1}, I^{n} \times\{0\}\right)$ and $\left(I^{n+1},\left(I^{n} \times\{0\}\right) \cup\left(\{0\} \times I^{n}\right)\right)$ are homeomorphic and $\mathcal{O}(p)$ is a Serre fibration, there exists a continuous map $G: I^{n+1} \rightarrow$ $\mathcal{O}(X)$ such that $G$ inc $=F$ and $\mathcal{O}(p) G=H q_{n+1}$. Since $G(0, z)=F(0, z)=x_{0}$ for all $z \in I^{n}$, there exists a continuous map $\bar{G}: \mathbb{I}^{n} I \rightarrow \mathcal{O}(X)$ such that $\bar{G} q_{n+1}=G$. The map $\bar{G}$ is the desired lift.

Proposition 4.3. Let $E$ and $B$ be topological spaces and let $p: E \rightarrow B$ be an A-fibration. Then $p$ has the homotopy lifting property with respect to the class of generalized $C W(A)$ complexes.

The proof follows by standard arguments applying the homeomorphism given in item (a) of 2.10

Note that if the core $A$ is the suspension of a locally compact and Hausdorff space then a map $p$ is an $A$-fibration if and only if it has the homotopy lifting property with respect to the spaces $A$ and $\mathrm{C} \Sigma^{n-1} A, n \in \mathbb{N}$, since in this case, by 2.9, $\mathbb{I}^{n} A$ is a $\mathrm{CW}(A)$-complex for all $n \in \mathbb{N}$.

We will prove now some results which will be needed for our purposes.

Proposition 4.4. Let $E, B$ topological spaces and let $p: E \rightarrow B$ be a continuous map. If $A$ is an $H$-cogroup, the following are equivalent

(a) $p \in \mathrm{Fib} \cap \mathrm{WE}$.

(b) The map $p$ has the $R L P$ with respect to the inclusion maps $\Sigma^{n-1} A \hookrightarrow \mathrm{C} \Sigma^{n-1} A$ $\forall n \in \mathbb{N}$ and with respect to the maps $* \rightarrow A$ and $i_{0}: \mathbb{I}^{n-1} A \rightarrow \mathbb{I}^{n} A \forall n \in \mathbb{N}$.

Moreover, any of these implies the following

(c) The map $p$ has $R L P$ with respect to the inclusion $B \hookrightarrow X$ for all generalized relative $C W(A)$-complexes $(X, B)$. 
Proof. (a) $\Rightarrow$ (b) We consider first a commutative diagram

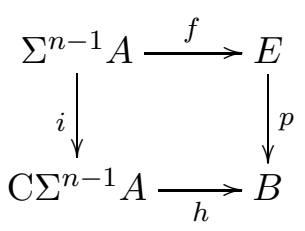

where $n \in \mathbb{N}$.

Since $p$ is an $A$-weak equivalence, from lemma 5.6 of [8] we get that there exists a continuous map $h^{\prime}: \mathrm{C} \Sigma^{n-1} A \rightarrow E$ such that $\left.h^{\prime}\right|_{\Sigma^{n-1} A}=f$ and $p h^{\prime} \simeq h$ rel $\Sigma^{n-1} A$. Let $H: \mathbb{I C} \Sigma^{n-1} A \rightarrow B$ be a homotopy from $p h^{\prime}$ to $h$ relative to $\Sigma^{n-1} A$. There is a commutative diagram

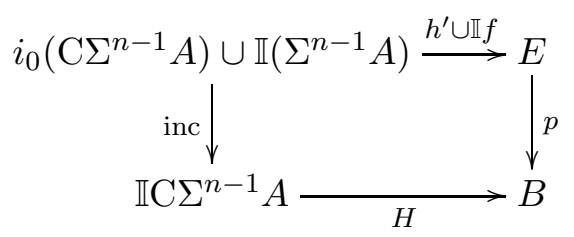

Since $p$ is an $A$-fibration, from item (a) of 2.10 we conclude that there exists a map $H^{\prime}: \mathbb{I C} \Sigma^{n-1} A \rightarrow E$ such that $p H^{\prime}=H$ and $H^{\prime}$ inc $=h^{\prime} \cup \mathbb{I} f$. The map $H^{\prime} i_{1}: \mathrm{C} \Sigma^{n-1} A \rightarrow E$ is the desired lift.

Consider now a commutative diagram

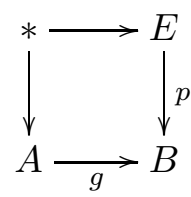

Since $p$ is an $A$-weak equivalence there exists a continuous map $\bar{g}: A \rightarrow E$ such that $p \bar{g} \simeq g$. Let $H: \mathbb{I} A \rightarrow B$ be a homotopy between $p \bar{g}$ and $g$. There is a commutative diagram

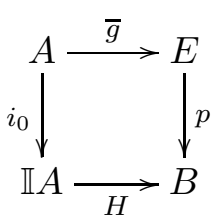

Since $p$ is an $A$-fibration there exists a continuous map $\bar{H}: \mathbb{I} A \rightarrow E$ such that $\bar{H} i_{0}=\bar{g}$ and $p \bar{H}=H$. Hence, $p \bar{H} i_{1}=H i_{1}=g$. Thus $\bar{H} i_{1}$ is the desired lift.

Finally, the RLP with respect to the maps $i_{0}: \mathbb{I}^{n-1} A \rightarrow \mathbb{I}^{n} A \forall n \in \mathbb{N}$ follows from the definition of $A$-fibration.

(b) $\Rightarrow$ (a) We will prove first that $p$ is an $A$-fibration. Let $b_{0}$ be the base point of $B$, let $n \in \mathbb{N}$ and let $f: C \Sigma^{n-1} A \rightarrow E$ and $H: I C \Sigma^{n-1} A \rightarrow B$ be continuous maps such that $H i_{0}=p f$.

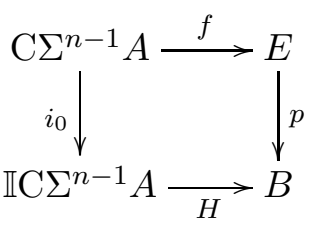


Let $G: \mathbb{I}\left(\mathbb{I} \Sigma^{n-1} A\right) \rightarrow B$ be defined by

$$
G(a, s, t)= \begin{cases}H\left(\left[\left[a, \frac{s}{1-t}\right], 2 t\right]\right) & \text { if } a \in \Sigma^{n-1} A, 0 \leq s \leq 1 \text { and } 0 \leq t \leq \min \left\{\frac{1}{2}, 1-s\right\} \\ H\left(\left[\left[a, \frac{s}{t}\right], 2(1-t)\right]\right) & \text { if } a \in \Sigma^{n-1} A, 0 \leq s \leq 1 \text { and } \max \left\{\frac{1}{2}, s\right\} \leq t \leq 1 \\ H([[a, 1], 2(1-s)]) & \text { if } a \in \Sigma^{n-1} A, 0 \leq s \leq 1 \text { and } 1-s \leq t \leq s\end{cases}
$$

It is easy to check that $G$ is well-defined and continuous. Moreover, since $G(a, 0, t)=b_{0}$ for all $a \in \Sigma^{n-1} A$ and $t \in[0,1]$, then there exists a map $\bar{G}: \mathbb{I C} \Sigma^{n-1} A \rightarrow B$ such that $\bar{G} q=G$, where $q: \mathbb{I}\left(\mathbb{I} \Sigma^{n-1} A\right) \rightarrow \mathbb{I C} \Sigma^{n-1} A$ is the quotient map.

Let $D=i_{0}\left(\mathrm{C} \Sigma^{n-1} A\right) \cup \mathbb{I} i\left(\Sigma^{n-1} A\right) \cup i_{1}\left(\mathrm{C} \Sigma^{n-1} A\right) \subseteq \mathbb{I C} \Sigma^{n-1} A$. Let $F: D \rightarrow B$ be defined by

$$
F(a, s, t)= \begin{cases}f([a, s]) & \text { if } a \in \Sigma^{n-1} A, 0 \leq s \leq 1 \text { and } t \in\{0,1\} \\ f([a, 1]) & \text { if } a \in \Sigma^{n-1} A, s=1 \text { and } 0 \leq t \leq 1\end{cases}
$$

It is easy to check that $F$ is well-defined and continuous. Moreover, there is a commutative diagram

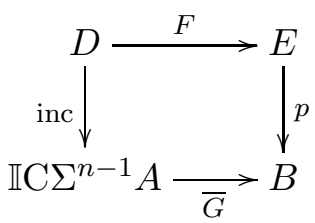

By hypothesis and applying item (b) of 2.10 we obtain that there exists a map $K$ : $\mathbb{I C} \Sigma^{n-1} A \rightarrow E$ such that $K$ inc $=F$ and $p K=\bar{G}$.

Let $k: \mathbb{I C} \Sigma^{n-1} A \rightarrow E$ be defined by

$$
k([[a, s], t])=K\left(\left[\left[a, s\left(1-\frac{t}{2}\right)\right], \frac{t}{2}\right]\right) .
$$

It is easy to check that $k i_{0}=f$ and $p k=H$.

The homotopy lifting properties with respect to $A$ and $\mathbb{I}^{n} A, n \in \mathbb{N}$, follow from the hypothesis.

Thus, $p$ is an $A$-fibration.

We will prove now that $p$ is an $A$-weak equivalence. We will prove first that $p$ induces isomorphisms $p_{*}: \pi_{n}^{A}(E) \rightarrow \pi_{n}^{A}(B)$ for $n \geq 1$.

Let $n \in \mathbb{N}$ and let $f, g: \Sigma^{n} A \rightarrow E$ be continuous maps such that $p f \simeq p g$. Let $H: \mathbb{I} \Sigma^{n} A \rightarrow B$ be the homotopy.

Let $q: \mathrm{C} \Sigma^{n-1} A \rightarrow \mathrm{C} \Sigma^{n-1} A / \Sigma^{n-1} A \cong \Sigma^{n} A$ be the quotient map. Hence $f q, g q$ : $\mathrm{C} \Sigma^{n-1} A \rightarrow E$ and

$$
H \circ \mathbb{I} q: \mathbb{I C} \Sigma^{n-1} A \rightarrow B
$$

is a homotopy between $p f q$ and $p g q$ relative to $\Sigma^{n-1} A$.

We consider in $\mathbb{I C} \Sigma^{n-1} A$ the following equivalence relation:

$$
(x, t) \sim\left(x^{\prime}, t^{\prime}\right) \Leftrightarrow x \in \Sigma^{n-1} A \text { and } x=x^{\prime}
$$

By 2.11 there exists a homeomorphism $\mathbb{I C} \Sigma^{n-1} A / \sim \cong \mathrm{C} \Sigma^{n} A$ which takes the classes of the elements of the bottom and the top of the cylinder to $\Sigma_{-} \Sigma^{n-1} A$ and $\Sigma_{+} \Sigma^{n-1} A$ respectively. Let $q^{\prime}: \mathbb{I C} \Sigma^{n-1} A \rightarrow \mathbb{I C} \Sigma^{n-1} A / \sim \cong \mathrm{C} \Sigma^{n} A$ be the quotient map. Since the homotopy $H \circ \mathbb{I} q$ is relative to $\Sigma^{n-1} A$, there exists a continuous map $H^{\prime}: \mathrm{C} \Sigma^{n} A \rightarrow B$ such that $H^{\prime} q^{\prime}=H \circ \mathbb{I} q$. 
Note that $\left.H^{\prime}\right|_{\Sigma^{n} A}$ equals $p f q$ in the southern hemisphere $\Sigma_{-} \Sigma^{n-1} A \cong \mathrm{C} \Sigma^{n-1} A$ and $p g q$ in the northern hemisphere $\Sigma_{+} \Sigma^{n-1} A \cong \mathrm{C} \Sigma^{n-1} A$. Hence, there is a commutative diagram

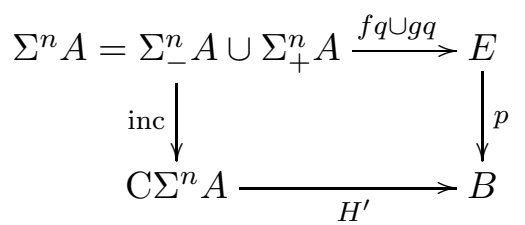

By hypothesis there exists a continuous map $K: \mathrm{C} \Sigma^{n} A \rightarrow E$ such that $p K=H^{\prime}$ and $\left.K\right|_{\Sigma^{n} A}=f q \cup g q$. Then $K q^{\prime}: \mathbb{I C} \Sigma^{n-1} A \rightarrow E, K q^{\prime} i_{0}=f q, K q^{\prime} i_{1}=g q$. Moreover, $K q^{\prime}$ is a homotopy relative to $\Sigma^{n-1} A$. Hence, if $s_{0}$ is the base point of $\mathrm{C} \Sigma^{n-1} A / \Sigma^{n-1} A \cong \Sigma^{n} A$ and $e_{0}$ is the base point of $E$, then for $s \in \Sigma^{n-1} A$ and $t \in I$ we have that $K q^{\prime}(s, t)=$ $K \operatorname{inc}\left(s, \frac{1}{2}\right)=f q(s)=f\left(s_{0}\right)=e_{0}$.

Then there exists a continuous map $K^{\prime \prime}: \mathbb{I} \Sigma^{n} A \rightarrow E$ such that $K^{\prime \prime} \circ \mathbb{I} q=K q^{\prime}$. Hence, $f q(x)=K q^{\prime} i_{0}(x)=K^{\prime \prime}\left(q \times \operatorname{Id}_{I}\right) i_{0}(x)=K^{\prime \prime}(q(x), 0)$. Since $q$ is surjective, $K^{\prime \prime} i_{0}=f$. In a similar way $K^{\prime \prime} i_{1}=g$, and hence $K^{\prime \prime}$ is a homotopy between $f$ and $g$. Thus, $p_{*}$ is injective.

We will prove now that $p_{*}$ is surjective. Let $[f] \in \pi_{n}^{A}(B)$. There is a commutative diagram

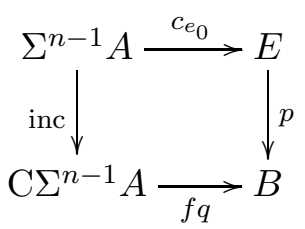

where $c_{e_{0}}$ is the constant map $e_{0}$. By hypothesis there exists $g: \mathrm{C} \Sigma^{n-1} A \rightarrow E$ such that $p g=f q$ and $\left.g\right|_{\Sigma^{n-1} A}=c_{e_{0}}$. Then, there exists $g^{\prime}: \Sigma^{n} A \rightarrow E$ such that $g^{\prime} q=g$. Hence, $p g^{\prime} q=p g=f q$ and since $q$ is surjective we obtain that $p g^{\prime}=f$. Thus, $p_{*}$ is surjective.

It remains to prove that $p_{*}: \pi_{0}^{A}(E) \rightarrow \pi_{0}^{A}(B)$ is an isomorphism. Note that $\pi_{0}^{A}(E)$ and $\pi_{0}^{A}(B)$ are groups since $A$ is an $H$-cogroup. This will be used to prove that $p_{*}$ is a monomorphism as we will show that $p f \simeq *$ implies $f \simeq *$.

Since $p$ has the RLP with respect to $* \rightarrow A$, it follows that $p_{*}$ is an epimorphism. Suppose now that $f: A \rightarrow E$ is a map such that $p f \simeq *$. Hence, there exists a map $g: \mathrm{C} A \rightarrow B$ such that $\left.g\right|_{A}=p f$.

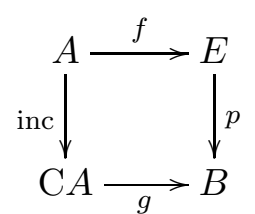

By hypothesis there exists a map $g^{\prime}: \mathrm{C} A \rightarrow E$ such that $p g^{\prime}=g$ and $g^{\prime} \mid A=f$. Hence, $f$ can be extended to $\mathrm{C} A$ and then $f \simeq *$.

(b) $\Rightarrow$ (c) follows by standard arguments.

From the previous proposition we obtain some interesting corollaries.

Corollary 4.5. If $A$ is an $H$-cogroup then $\mathcal{I}_{A \text {-inj }}=\mathcal{J}_{A}$-inj $\cap \mathrm{WE}$.

Corollary 4.6. If $A$ is an $H$-cogroup then every generalized $C W(A)$-complex is an $A$ cofibrant space.

Corollary 4.7. If $A$ is an $H$-cogroup then the inclusion maps $* \rightarrow A, \Sigma^{n-1} A \hookrightarrow \mathrm{C} \Sigma^{n-1} A$, $n \in \mathbb{N}$, and $i_{0}: \mathbb{I}^{n-1} A \rightarrow \mathbb{I}^{n} A, n \in \mathbb{N}$, are $A$-cofibrations. 
Remark 4.8. Proposition 4.4 also holds if $A=S^{0}$ and $E$ is path-connected. Note that the hypothesis of $A$ being an $H$-cogroup is only used to prove injectivity of the map $p_{*}: \pi_{0}^{A}(E) \rightarrow \pi_{0}^{A}(B)$ which follows trivially if $A=S^{0}$ and $E$ is path-connected.

However, this does not hold if the space $E$ is not path-connected as the following example shows. Let $E=\{a, b, c\}$ with the discrete topology and with base point $a$, let $B=S^{0}$ and let $p: E \rightarrow B$ be defined by $p(a)=1, p(b)=-1, p(c)=-1$. It is easy to verify that $p$ satisfies part (b) of proposition 4.4 but it does not satisfy part (a) since it does not induce an isomorphism $p_{*}: \pi_{0}^{S^{0}}(E) \rightarrow \pi_{0}^{S^{0}}(B)$.

If $X$ is a topological space, $X^{I}$ will denote the space of continuous maps from $I$ to $X$ which do not necessarily preserve base points. We give $X^{I}$ the compact-open topology. If $x_{0}$ is the base point of $X$, the constant map from $I$ to $X$ with value $x_{0}$ will be the base point of $X^{I}$. We define the maps $\mathrm{ev}_{0}^{X}, \mathrm{ev}_{1}^{X}: X^{I} \rightarrow X$ by $\mathrm{ev}_{0}^{X}(\alpha)=\alpha(0)$ and $\mathrm{ev}_{1}^{X}(\alpha)=\alpha(1)$. Also, if $f: X \rightarrow Y$ is a continuous map, we define $f^{I}: X^{I} \rightarrow Y^{I}$ by $f^{I}(\alpha)=f \circ \alpha$.

If $f: \mathbb{I} X \rightarrow Y$ is a continuous map, $f^{\sharp}$ will denote the continuous map from $X$ to $Y^{I}$ defined by the exponential law. Also, if $g: X \rightarrow Y^{I}$ is a continuous map, $g^{b}$ will denote the continuous map from $\mathbb{I} X$ to $Y$ defined by the exponential law.

Lemma 4.9. Let $X$ and $Y$ be topological spaces, let $p: X \rightarrow Y$ be an A-fibration and let $X \underset{Y}{\times} Y^{I}$ be the pullback

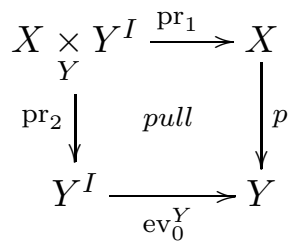

If $A$ is an H-cogroup, the map $\left(\mathrm{ev}_{0}^{X}, p^{I}\right): X^{I} \rightarrow \underset{Y}{X} \underset{Y}{\times} Y^{I}$ is a trivial A-fibration.

Proof. Suppose that there is a commutative diagram

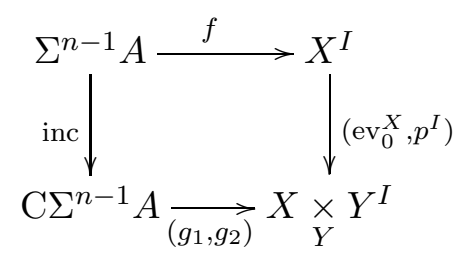

with $n \in \mathbb{N}$. Then, there is a commutative diagram

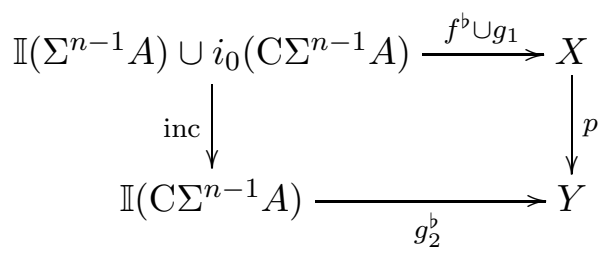

Since $p$ is an $A$-fibration, by item (a) of 2.10 there exists a map $H^{\prime}: \mathbb{I C} \Sigma^{n-1} A \rightarrow X$ such that $p H^{\prime}=g_{2}^{b}$ and $H^{\prime}$ inc $=f^{b} \cup g_{1}$. Thus, by the exponential law we obtain a continuous map $H: \mathrm{C} \Sigma^{n-1} A \rightarrow X^{I}$ and $H$ is the desired lift. 
Suppose now that there is a commutative diagram

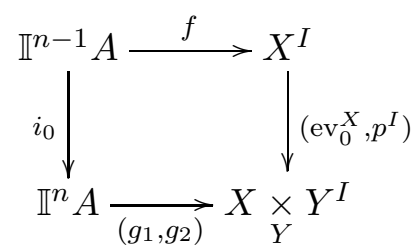

with $n \in \mathbb{N}$. Applying the exponential law we obtain a commutative diagram

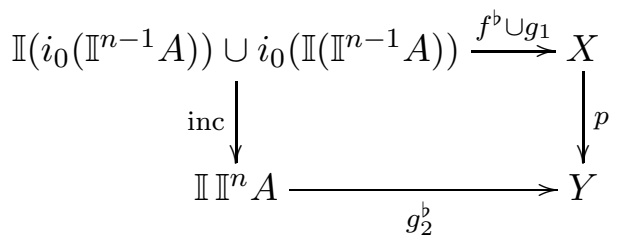

Since $p$ is an $A$-fibration, by item (c) of lemma 2.10 there exists a map $H^{\prime}: \mathbb{I} \mathbb{I}^{n} A \rightarrow X$ such that $H^{\prime}$ inc $=f^{b} \cup g_{1}$ and $p H^{\prime}=g_{2}^{b}$. Now, from $H^{\prime}$ and the exponential law we get the desired lift $H: \mathbb{I}^{n} A \rightarrow X^{I}$.

The right lifting property with respect to $* \rightarrow A$ can be proved in a similar way.

Thus, by 4.4 , the map $\left(\mathrm{ev}_{0}^{X}, p^{I}\right)$ is a trivial $A$-fibration.

Definition 4.10. Let $p: X \rightarrow Y$ be a continuous map between pointed topological spaces. We say that $p$ is a pointed Hurewicz fibration if $p$ is a pointed map which has the homotopy lifting property (in the category of pointed topological spaces) with respect to any pointed space.

If $Z$ is a topological space we define the map $s_{Z}: Z \rightarrow Z^{I}$ by $s_{Z}(z)(t)=z$ for $z \in Z$ and $t \in I$.

Proposition 4.11. Let $B$ and $Z$ be topological spaces and let $i: B \rightarrow Z$ be a continuous map. If $A$ is an H-cogroup, the following are equivalent:

(a) $i \in \mathrm{Cof} \cap \mathrm{WE}$.

(b) $i$ has the LLP with respect to Fib.

(c) $i \in$ Cof and $i$ is a strong deformation retract.

Proof. (a) $\Rightarrow$ (c) We consider the usual factorization $i=p j$ where $p$ is a Hurewicz fibration and $j$ is a strong deformation retract. It is easy to verify that $p$ is a pointed Hurewicz fibration and hence $p \in$ Fib. On the other hand, $j$ is an $A$-weak equivalence, and since $i$ is also an $A$-weak equivalence it follows that $p \in \mathrm{WE}$. Hence, $p \in \mathrm{Fib} \cap \mathrm{WE}$. Since $i \in \mathrm{Cof}$, there exists a lift $u$ such that the following diagram commutes.

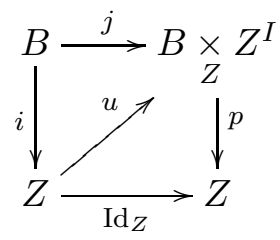

Thus, $i$ is a retract of $j$ and it follows that $i$ is also a strong deformation retract.

(c) $\Rightarrow$ (a) Follows immediately. 
(b) $\Rightarrow$ (c) Clearly $i \in$ Cof. Also, since the map $B \rightarrow *$ is a pointed Hurewicz fibration, it is an $A$-fibration. Hence, there exists a map $r$ such that the following diagram commutes:

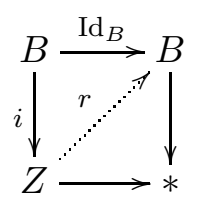

Then $r i=\operatorname{Id}_{B}$.

Also, it is easy to prove that the map $\left(\mathrm{ev}_{0}^{Z}, \mathrm{ev}_{1}^{Z}\right): Z^{I} \rightarrow Z \times Z$ is a pointed Hurewicz fibration. Hence it is an $A$-fibration.

By hypothesis, there exists a map $H$ such that the following diagram commutes:

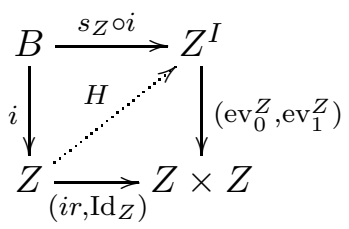

Then, $H^{\mathrm{b}}: i r \simeq \operatorname{Id}_{Z} \operatorname{rel}(i(B))$. Thus, $i$ is a strong deformation retract.

(c) $\Rightarrow$ (b) Let $p: X \rightarrow Y$ be an $A$-fibration and suppose there is a commutative diagram

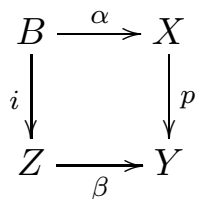

Since $i$ is a strong deformation retract, there exist $r: Z \rightarrow B$ and $h: I Z \rightarrow Z$ such that $r i=\operatorname{Id}_{B}$ and $h: i r \simeq \operatorname{Id}_{Z} \operatorname{rel}(i(B))$. By 4.9, the map $\left(\operatorname{ev}_{0}^{X}, p^{I}\right): X^{I} \rightarrow X \underset{Y}{\times} Y^{I}$ is a trivial $A$-fibration and since $i \in$ Cof and

$$
\begin{aligned}
\left(\mathrm{ev}_{0}^{X}, p^{I}\right) s_{X} \alpha & =\left(\operatorname{ev}_{0}^{X} s_{X} \alpha, p^{I} s_{X} \alpha\right)=\left(\alpha, s_{Y} p \alpha\right)=\left(\alpha, s_{Y} \beta i\right)=\left(\alpha, \beta^{I} s^{Z} i\right)= \\
& =\left(\alpha r i, \beta^{I} h^{\sharp} i\right)=\left(\alpha r, \beta^{I} h^{\sharp}\right) i
\end{aligned}
$$

there exists a continuous map $H$ that such that the following diagram commutes

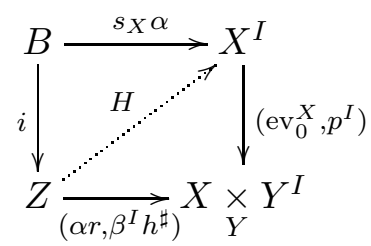

Let $u=\operatorname{ev}_{1}^{X} H$. Then

$$
p u=p \operatorname{ev}_{1}^{X} H=\operatorname{ev}_{1}^{Y} p^{I} H=\operatorname{ev}_{1}^{Y} \beta^{I} h^{\sharp}=\beta \operatorname{ev}_{1}^{Z} h^{\sharp}=\beta h i_{1}=\beta
$$

and

$$
u i=\mathrm{ev}_{1}^{X} H i=\mathrm{ev}_{1}^{X} s_{X} \alpha=\alpha .
$$

Then, $u$ is the desired lift. Thus $i$ has the LLP with respect to Fib.

Corollary 4.12. If $A$ is an $H$-cogroup then $\mathcal{J}_{A}$-cof $=\mathcal{I}_{A}$-cof $\cap \mathrm{WE}$. 
Proof. Note that $\mathcal{J}_{A}$-cof is the class of maps that have the LLP with respect to the class Fib since Fib $=\mathcal{J}_{A}$-inj. On the other hand, $\mathcal{I}_{A}$-cof is the class of maps that have the LLP with respect to the class Fib $\cap \mathrm{WE}$ since $\mathcal{I}_{A^{-i n j}}=\mathcal{J}_{A}$-inj $\cap \mathrm{WE}=$ Fib $\cap \mathrm{WE}$ by 4.5. Hence, $\mathcal{I}_{A}$-cof $=$ Cof. The result follows from 4.11

Theorem 4.13. Let $A$ be a compact and $T_{1}$ topological $H$-cogroup. Then the category of topological spaces with $\mathrm{Fib}$, Cof and $\mathrm{WE}$ as the classes of fibrations, cofibrations and weak equivalences respectively is a cofibrantly generated model category.

Proof. Note that by 4.4 a map belongs to Fib $\cap$ WE if and only if it has the RLP with respect to the class $\mathcal{I}_{A}$. Also, by definition, a map is a fibration if and only if it has the RLP with respect to the class $\mathcal{J}_{A}$. Hence, by theorem 11.3.1 of [5], it suffices to prove that

(0) WE is closed under retracts and satisfies the 'two out of three' axiom.

(1) Both $\mathcal{I}_{A}$ and $\mathcal{J}_{A}$ permit the small object argument.

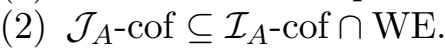

(3) $\mathcal{I}_{A}$-inj $\subseteq \mathcal{J}_{A}$-inj $\cap \mathrm{WE}$.

(4) $\mathcal{I}_{A^{-}}$-cof $\cap \mathrm{WE} \subseteq \mathcal{J}_{A^{-} \text {-cof or }} \mathcal{J}_{A^{-}}$-inj $\cap \mathrm{WE} \subseteq \mathcal{I}_{A^{-}}$-inj.

The proof of (0) is straightforward.

To prove (1) note that the maps in $\mathcal{I}_{A}$ or $\mathcal{J}_{A}$ are closed inclusions into $\mathrm{T}_{1}$ spaces since $A$ is a $\mathrm{T}_{1}$ space. Also, the domains of those maps are compact spaces since $A$ is a compact space. Since compact topological spaces are finite relative to closed $\mathrm{T}_{1}$ inclusions ([6], proposition 2.4.2) we obtain that $\mathcal{I}_{A}$ and $\mathcal{J}_{A}$ permit the small object argument.

Items (2), (3) and (4) follow from 4.5 and 4.12.

Remark 4.14. Note that this model category structure is finitely generated. Note also that, with this model category structure, every topological space is fibrant and every generalized $\mathrm{CW}(A)$-complex is cofibrant.

Remark 4.15. Theorem 4.13 also holds for $A=S^{0}$ if we work in the category of pathconnected topological spaces since proposition 4.4 also holds under these hypothesis (cf. remark 4.8). More precisely, the category of pointed and path-connected topological spaces with the classes of $S^{0}$-fibrations, $S^{0}$-cofibrations and $S^{0}$-weak equivalences as the classes of fibrations, cofibrations and weak equivalences respectively is a cofibrantly generated model category.

Moreover, this model category structure coincides with the usual model category structure in the category of pointed topological spaces restricted to the full subcategory of path-connected spaces.

As a consequence of the small object argument ([5], proposition 10.5.16) we obtain the following $\mathrm{CW}(A)$-approximation theorem.

Theorem 4.16. Let $A$ be a compact and $T_{1}$ topological H-cogroup. Let $X$ be a topological space. Then there exists a generalized $C W(A)$-complex $Z$ together with an $A$-weak equivalence $f: Z \rightarrow X$.

Proof. Since the class $\mathcal{I}_{A}$ permits the small object argument we can factorize the map $* \rightarrow X$ into a relative $\mathcal{I}_{A}$-cell complex $i: * \rightarrow W$ followed by an $\mathcal{I}_{A}$-injective $p: W \rightarrow X$ (note that $p$ is an $A$-weak equivalence by 4.5 ). Moreover, in this factorization we only need to take a countably infinite sequence of pushouts since the domains of the maps of $\mathcal{I}_{A}$ are finite relative to $\mathcal{I}_{A}$ as we pointed out in the previous proof. 
Thus, the space $W$ is constructed in much the same way as a generalized $\mathrm{CW}(A)$ complex, but some 'extra' cells are attached taking pushouts with maps $\mathbb{I}^{k-1} A \rightarrow \mathbb{I}^{k} A$. However, since the inclusion $\mathbb{I}^{k-1} A \hookrightarrow \mathbb{I}^{k} A$ is a strong deformation retract, we can replace each step $W_{n}$ of the construction of $W$ by a generalized $\mathrm{CW}(A)$-complex $Z_{n}$ constructed from $Z_{n-1}$ by attaching the same cells that are attached to $W_{n-1}$, excluding those coming from maps $\mathbb{I}^{k-1} A \rightarrow \mathbb{I}^{k} A$. By a similar reasoning as the one in the proof of theorem 4.1 of 8 we obtain that $Z_{n}$ is a strong deformation retract of $W_{n}$ and if we define $Z$ as the colimit of the spaces $Z_{n}, n \in \mathbb{N}$, we get that $Z$ is a generalized $\mathrm{CW}(A)$-complex and a strong deformation retract of $W$. Composing the strong deformation retract $j: Z \rightarrow W$ with the map $p$ we get an $A$-weak equivalence $f: Z \rightarrow X$.

Now, we will apply the model category structure defined above to obtain an $A$-based version of Whitehead's theorem. To this end, we need the following proposition which relates Quillen's left homotopies with usual homotopies.

Proposition 4.17. Let $A$ be a compact and $T_{1}$ topological $H$-cogroup. Let $X$ be an $A$ cofibrant space and let $Y$ be another topological space. Let $g_{1}, g_{2}: X \rightarrow Y$ be continuous maps. Then $g_{1}$ and $g_{2}$ are homotopic (in the usual sense) if and only if they are left homotopic (in the sense of Quillen).

Proof. As usual, if $g_{1}$ and $g_{2}$ are homotopic, factorizing the map $i_{0}+i_{1}: X \vee X \rightarrow \mathbb{I} X$ into an $A$-cofibration followed by an $A$-weak equivalence we obtain a cylinder object $X^{\prime}$ and a left homotopy $H: X^{\prime} \rightarrow Y$ between $g_{1}$ and $g_{2}$.

Suppose now that $g_{1}$ and $g_{2}$ are left homotopic. Note that $Y^{I}$ is a path object for $Y$ since the map $\left(\mathrm{ev}_{0}, \mathrm{ev}_{1}\right): Y^{I} \rightarrow Y \times Y$ is a pointed Hurewicz fibration and the map $s_{Y}: Y \rightarrow Y^{I}$ (which was defined by $s_{Y}(y)(t)=y$ for $y \in Y$ and $t \in I$ ) is a homotopy equivalence. Since $X$ is $A$-cofibrant and $Y^{I}$ is a path object for $Y$ then by proposition 1.2.5 of [6] there exists a right homotopy $K: X \rightarrow Y^{I}$ between $g_{1}$ and $g_{2}$. Applying the exponential law we get that $g_{1}$ and $g_{2}$ are homotopic in the usual sense.

The following result is the $A$-based version of Whitehead's theorem mentioned before. It generalizes theorem 5.10 of $[8]$ and is related to theorem E.1 of chapter 2 of [3].

Theorem 4.18. Let $A$ be a compact and $T_{1}$ topological $H$-cogroup. Let $X$ and $Y$ be topological spaces and let $f: X \rightarrow Y$ be an $A$-weak equivalence. If $X$ and $Y$ are $A$ cofibrant spaces then $f$ is a homotopy equivalence. In particular, this holds if $X$ and $Y$ are generalized $C W(A)$-complexes.

Proof. The result follows by standard arguments applying the previous proposition and the fact that every space is fibrant in this model category structure (cf. proposition 1.2.8 of $[6])$.

\section{Quillen FunCtors}

In this section we will give three Quillen adjunctions related to the $A$-based cofibrantly generated model category structure developed before. The first of them shows that, under certain hypotheses, the exponential law is a Quillen adjunction. The second and third ones relate the model category structure defined in the previous section for different choices of the base space $A$. Namely, we give adjunctions between an $A$-based and a $B$-based structure when the space $B$ is a retract of $A$ and when the space $B$ is an iterated suspension of $A$. 
From now on, if $A$ is a compact and $\mathrm{T}_{1}$ topological $H$-cogroup, $\mathcal{T}_{o} p_{A}$ will denote the model category of pointed topological spaces with the $A$-based cofibrantly generated model category structure defined in the previous section.

Lemma 5.1. Let $E$ and $B$ be topological spaces and let $p: E \rightarrow B$ be a continuous map. If $p$ is an $S^{0}$-fibration then $p$ is an $S^{1}$-fibration.

Proof. We have to prove that the map $p$ has the homotopy lifting property with respect to $\mathbb{I}^{n} S^{1}$ for all $n \in \mathbb{N}_{0}$. Let $n \in \mathbb{N}_{0}$ and let $f: \mathbb{I}^{n} S^{1} \rightarrow E$ and $H: \mathbb{I}\left(\mathbb{I}^{n} S^{1}\right) \rightarrow B$ be continuous maps such that $H i_{0}=p f$. For $j \in \mathbb{N}$ let $q_{j}: I^{j} \rightarrow I^{j} /\left(\{0,1\} \times I^{j-1}\right)$ be the quotient map and let $\varphi_{j}: I^{j} /\left(\{0,1\} \times I^{j-1}\right) \rightarrow \mathbb{I}^{j-1} S^{1}$ be the homeomorphism defined by $\varphi\left[\left(t_{1}, t_{2}, \ldots, t_{j}\right)\right]=\left[\left(e^{2 \pi t_{1} i}, t_{2}, \ldots, t_{j}\right)\right]$.

There is a commutative diagram of pointed spaces and maps

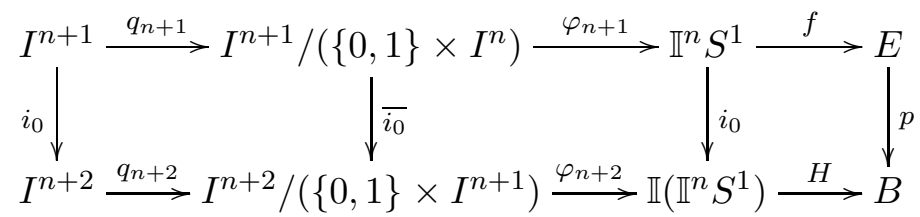

where $\overline{i_{0}}$ is the map that makes commutative the left square.

Let $e_{0}$ be the base point of $E$. Consider the following diagram in the category of (unbased) topological spaces.

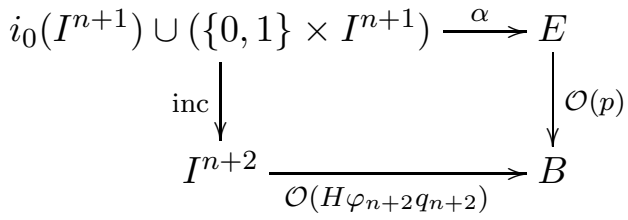

where $\alpha$ is defined by $\alpha(x, 0)=f \varphi_{n+1} q_{n+1}(x)$ for all $x \in I^{n+1}$ and $\alpha(t, y)=e_{0}$ for all $t \in\{0,1\}$ and $y \in I^{n+1}$. Note that $\alpha$ is well defined and continuous. Note also that the previous diagram is commutative.

Since $p$ is an $S^{0}$-fibration then, by 4.2, $\mathcal{O}(p)$ is a Serre fibration. Hence, there exists a continuous map $H^{\prime}: I^{n+2} \rightarrow E$ such that $H^{\prime}$ inc $=\alpha$ and $\mathcal{O}(p) H^{\prime}=\mathcal{O}\left(H \varphi_{n+2} q_{n+2}\right)$. Thus, $H^{\prime}(t, y)=e_{0}$ for all $t \in\{0,1\}$ and $y \in I^{n+1}$. Then, there exists a continuous map $\overline{H^{\prime}}: I^{n+2} /\left(\{0,1\} \times I^{n+1}\right) \rightarrow E$ such that $\overline{H^{\prime}} q_{n+2}=H^{\prime}$.

Since $\overline{H^{\prime}}$ preserves base points it can be seen as a map in the category of pointed topological spaces. Thus, let $K=\overline{H^{\prime}} \circ\left(\varphi_{n+2}\right)^{-1}: \mathbb{I}\left(\mathbb{I}^{n} S^{1}\right) \rightarrow E$. It is easy to prove that $K i_{0}=f$ and $p K=H$.

Proposition 5.2. Let $A$ be a compact and Hausdorff topological $H$-cogroup. Let $L$ : $\mathcal{T}_{o p} p_{S^{1}} \rightarrow \mathcal{T}_{o} p_{A}$ be the functor $\ldots \wedge A$ and let $R: \mathcal{T}_{o p_{A}} \rightarrow \mathcal{T}_{o p_{S^{1}}}$ be the functor $\operatorname{Hom}(A, \ldots)$. Let $\varphi$ be the natural isomorphism expressing $R$ as a right adjoint of $L$. Then $(L, R, \varphi)$ is a Quillen adjunction.

Proof. It suffices to prove that $R$ is a right Quillen functor. First, we will prove that $R$ preserves fibrations. Let $p: X \rightarrow Y$ be an $A$-fibration. Let $n \in \mathbb{N}$ and suppose we have a 
commutative diagram

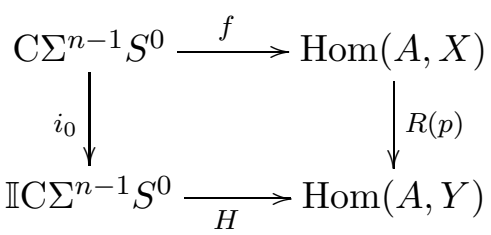

Then, by naturality of $\varphi$, there is a commutative diagram

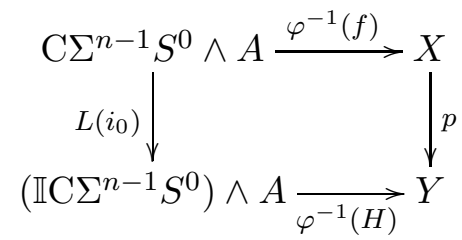

Since

$$
\left(\mathrm{C} \Sigma^{n-1} S^{0}\right) \wedge A=I \wedge S^{n-1} \wedge S^{0} \wedge A=\mathrm{C} \Sigma^{n-1} A
$$

and

$$
\left(\mathbb{I C} \Sigma^{n-1} S^{0}\right) \wedge A=I_{+} \wedge I \wedge S^{n-1} \wedge S^{0} \wedge A=\mathbb{I C} \Sigma^{n-1} A
$$

and $p$ is an $A$-fibration, there exists a continuous map $\widetilde{H}:\left(\mathbb{I C} \Sigma^{n-1} S^{0}\right) \wedge A \rightarrow X$ such that $\widetilde{H} \circ L\left(i_{0}\right)=\varphi^{-1}(f)$ and $p \circ \widetilde{H}=\varphi^{-1}(H)$. The map $\varphi(\widetilde{H}): \mathbb{I C} \Sigma^{n-1} S^{0} \rightarrow \operatorname{Hom}(A, X)$ is the desired lift.

In a similar way one can prove that the map $R(p)$ has the HLP with respect to $S^{0}$ and with respect to $\mathbb{I}^{n} S^{0}$ for all $n \in \mathbb{N}$. Hence $R(p)$ is an $S^{0}$-fibration. Thus, by the previous lemma, $R(p)$ is an $S^{1}$-fibration.

We will prove now that $R$ preserves weak equivalences. Let $g: X \rightarrow Y$ be a continuous map and let $n \in \mathbb{N}_{0}$. By the exponential law, there is a commutative diagram

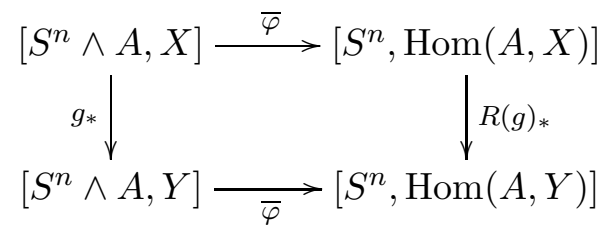

where $\bar{\varphi}$ is the natural isomorphism induced by $\varphi$. Since $S^{n} \wedge A=\Sigma^{n} A$, from the commutativity of the previous diagram it follows that if $g$ is an $A$-weak equivalence then $R(g): \operatorname{Hom}(A, X) \rightarrow \operatorname{Hom}(A, Y)$ is an $S^{0}$-weak equivalence, and hence it is an $S^{1}$-weak equivalence.

Thus, $R$ is a right Quillen functor.

Remark 5.3. Although the functor $R$ of the previous proposition takes $A$-weak equivalences to $S^{0}$-weak equivalences and $A$-fibrations to $S^{0}$-fibrations it can not be regarded as a Quillen functor to $\mathcal{T}_{0} p_{S^{0}}$ since $\mathcal{T}_{0} p_{S^{0}}$ is not a Quillen model category with our structure (cf. remark 4.15).

Now we turn our attention to the second adjunction mentioned at the beginning of this section which is essentially given by the following proposition.

Proposition 5.4. Let $A$ and $B$ be (pointed) topological spaces and let $f$ be a continuous map.

(1) If $B$ is a retract of $A$ and $f$ is an A-fibration then $f$ is a $B$-fibration. 
(2) If there exist continuous maps $\alpha: A \rightarrow B$ and $\beta: B \rightarrow A$ such that $\alpha \circ \beta \simeq \operatorname{Id}_{B}$ and $f$ is an $A$-weak equivalence then $f$ is a $B$-weak equivalence.

Proof. (1) If $B$ is a retract of $A$ then the maps $i_{0}: B \rightarrow \mathbb{I} B, i_{0}: \mathrm{C} \Sigma^{n-1} B \rightarrow \mathbb{C C} \Sigma^{n-1} B$, $n \in \mathbb{N}$, and $i_{0}: \mathbb{I}^{n-1} B \rightarrow \mathbb{I}^{n} B, n \in \mathbb{N}$, are retracts of the maps $i_{0}: A \rightarrow \mathbb{I} A, i_{0}: \mathrm{C} \Sigma^{n-1} A \rightarrow$ $\mathbb{I C} \Sigma^{n-1} A, n \in \mathbb{N}$, and $i_{0}: \mathbb{I}^{n-1} A \rightarrow \mathbb{I}^{n} A, n \in \mathbb{N}$, respectively. If $f$ is an $A$-fibration, $f$ has the RLP with respect to this last collection of maps and hence $f$ has the RLP with respect to the first collection of maps. Thus $f$ is a $B$-fibration.

(2) For each $n \in \mathbb{N}_{0}$ there is a commutative diagram

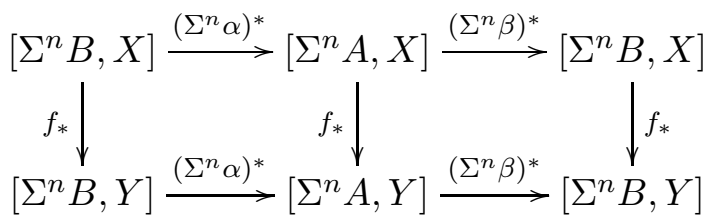

Since the horizontal compositions are the identity maps, the map $f_{*}:\left[\Sigma^{n} B, X\right] \rightarrow\left[\Sigma^{n} B, Y\right]$ is a retract of the map $f_{*}:\left[\Sigma^{n} A, X\right] \rightarrow\left[\Sigma^{n} A, Y\right]$ which is an isomorphism by hypothesis. Then $f_{*}:\left[\Sigma^{n} B, X\right] \rightarrow\left[\Sigma^{n} B, Y\right]$ is an isomorphism for all $n \in \mathbb{N}_{0}$ and hence $f$ is a $B$-weak equivalence.

Proposition 5.5. Let $A$ and $B$ be compact and $T_{1}$ topological $H$-cogroups. Let $L: \mathcal{T}_{o} p_{B} \rightarrow$ $\mathcal{T}_{o} p_{A}$ and $R: \mathcal{T}_{o} p_{A} \rightarrow \mathcal{T}_{o} p_{B}$ be the identity functors. Let $\phi$ be the natural isomorphism defined by the identity maps in all objects. If $B$ is a retract of $A$ then $(L, R, \phi)$ is a Quillen adjunction.

Proof. It suffices to prove that $R$ is a right Quillen functor, which follows from the previous proposition.

Clearly, if $B$ is a retract of $A$ and $A$ and $B$ are homotopy equivalent then the adjunction of above turns out to be a Quillen equivalence.

Now we turn our attention to the third adjunction which deals with suspensions.

Proposition 5.6. Let $A$ be a (pointed) topological space, let $n \in \mathbb{N}$ and let $f$ be a continuous map. If $A$ is the suspension of a locally compact and Hausdorff space and $f$ is an $A$-fibration then $f$ is a $\Sigma^{n} A$-fibration.

Proof. It is clear that for all $m \in \mathbb{N}, f$ has the homotopy lifting property with respect to the spaces $\mathrm{C} \Sigma^{m-1}\left(\Sigma^{n} A\right)$ since $f$ is an $A$-fibration. It remains to prove that $f$ has the homotopy lifting property with respect to the spaces $\mathbb{I}^{m} \Sigma^{n} A$ for all $m \in \mathbb{N}_{0}$.

Let $m \in \mathbb{N}_{0}$. By 2.9. $\left(\mathbb{I}\left(\mathbb{I}^{m} \Sigma^{n} A\right), i_{0}\left(\mathbb{I}^{m} \Sigma^{n} A\right)\right)$ is a relative $\mathrm{CW}(A)$-complex. Then, by 4.4 the map $i_{0}: \mathbb{I}^{m} \sum^{n} A \rightarrow \mathbb{I}\left(\mathbb{I}^{m} \sum^{n} A\right)$ has the LLP with respect to the trivial $A$-fibrations and hence it is an $A$-cofibration. But since it is also a homotopy equivalence, from 4.11 it follows that it has the LLP with respect to the $A$-fibrations. Hence, $f$ has the homotopy lifting property with respect to the space $\mathbb{I}^{m} \Sigma^{n} A$.

Since every $A$-weak equivalence is a $\Sigma^{n} A$-weak equivalence, from the previous proposition we obtain the following.

Proposition 5.7. Let $A$ be the suspension of a compact and Hausdorff space and let $n \in \mathbb{N}$. Let $L: \mathcal{T}_{o} p_{\Sigma^{n} A} \rightarrow \mathcal{T}_{o} p_{A}$ and $R: \mathcal{T}_{o} p_{A} \rightarrow \mathcal{T}_{o p_{\Sigma^{n} A}}$ be the identity functors. Let $\phi$ be the natural isomorphism defined by the identity maps in all objects. Then $(L, R, \phi)$ is a Quillen adjunction. 
If, in addition, $A$ is homotopy equivalent to a compact and $(k-1)$-connected CWcomplex of dimension $k$ for some $k \in \mathbb{N}$, then the adjunction of the previous proposition induces an equivalence of categories between $\mathrm{Ho}_{0} \mathcal{T}_{\Sigma^{n} A_{A}}$ and the full subcategory of Ho $\mathcal{T}_{0} p_{A}$ whose objects are the $A$ - $(n-1)$-connected spaces. The proof of this fact follows from theorem 3.2 of 2 in the following way. If $k \geq 2$, from homology decomposition (cf. theorem $4 \mathrm{H} .3$ of [4) it follows that a $(k-1)$-connected $\mathrm{CW}$-complex of dimension $k$ is homotopy equivalent to a wegde of spheres of dimension $k$. Note that this also holds for $k=1$.

Then, if $n \in \mathbb{N}$ an $A$-cofibrant space is $(n+k-1)$-connected if and only if it is $A$ $(n-1)$-connected. Indeed, the first implication is clear while the converse can be proved in a similar way to the first item of proposition 3.1 of [2].

On the other hand, if $B$ is a wegde of spheres of dimension $k$ and $X$ is a pointed space then $\pi_{n}^{B}(X)$ is a direct product of copies of the group $\pi_{n+k}(X)$. Hence a map is a $B$-weak equivalence if and only if it induces isomorphisms in $\pi_{m}$ for $m \geq k$. Thus, under the assumptions on the space $A$, a map is an $A$-weak equivalence if and only if it induces isomorphisms in $\pi_{m}$ for $m \geq k$.

Hence, applying theorem 3.2 of [2] one gets an equivalence of categories

$$
\begin{aligned}
\mathrm{Ho}_{\mathcal{T}} \mathcal{T}_{\Sigma^{n} A} & \left.\left.\cong \mathrm{Ho}_{o p}\right|_{(n+k-1)-\mathrm{conn}} \cong\left(\left.\operatorname{Ho} \mathcal{T}_{o p}\right|_{(k-1)-\mathrm{conn}}\right)\right|_{(n+k-1)-\mathrm{conn}} \cong \\
& \left.\cong \mathrm{Ho} \mathcal{T}_{0} p_{A}\right|_{A-(n-1)-\mathrm{conn}}
\end{aligned}
$$

where, for a category $\mathscr{C}$ and for $l \in \mathbb{N}_{0},\left.\mathscr{C}\right|_{l-\text { conn }}$ and $\left.\mathscr{C}\right|_{A-l-\text { conn }}$ denote the full subcategories of $\mathscr{C}$ whose objects are the $l$-connected spaces and the $A$-l-connected spaces respectively.

Note that although the model category developed in [2] is different from ours, their weak $n$-equivalences coincide with our $S^{n}$-weak equivalences and hence the homotopy category they obtain by inverting the weak $n$-equivalences is the same as Ho $\mathcal{T}_{o} p_{S^{n}}$.

\section{REFERENCES}

[1] Baues, J. Algebraic homotopy. Cambridge studies in advanced mathematics 15 (1989).

[2] Extremiana, J.; Hernández, L. and Rivas, M. A closed model category for $(n-1)$-connected spaces, Proceedings of the American Mathematical Society 124 (1996), 3545-3553.

[3] Farjoun, E. Cellular spaces, null spaces and homotopy localization. Lecture Notes in Mathematics 1622. Springer. 1995.

[4] Hatcher, Allen. Algebraic Topology. Cambridge University Press. 2002.

[5] Hirschhorn, P. Model categories and their localizations. Mathematical surveys and monographs, vol. 99. American Mathematical Society. 2003.

[6] Hovey, M. Model categories. Mathematical surveys and monographs, vol. 63. American Mathematical Society. 1999.

[7] Minian, G. Complexes in Cat. Topology and its Applications 119 (2002), 41-51.

[8] Minian, G. and Ottina, M. A geometric decomposition of spaces into cells of different types. Journal of Homotopy and Related Structures 1(1) (2006), 245-271.

[9] Minian, G. and Ottina, M. A geometric decomposition of spaces into cells of different types II: Homology Theory. Topology and its Applications 155 (2008), 1777-1785.

[10] Ottina, M. Different Types of Cellular Structures in Topological Spaces. Ph.D. Thesis. 2009.

[11] Quillen, D. Homotopical Algebra. Lecture Notes in Mathematics. Vol. 43. Springer. 1967.

[12] Whitehead, J.H.C. Combinatorial Homotopy I. Bulletin of the American Mathematical Society 55 (1949), 213-245.

E-mail address: emottina@uncu.edu.ar

Facultad de Ciencias Exactas y Naturales, Universidad Nacional de Cuyo, Mendoza, ArGENTINA. 